\title{
NEWMAN'S INEQUALITY FOR INCREASING EXPONENTIAL SUMS
}

\author{
TAMÁS ERDÉLYI
}

Dedicated to the memory of George G. Lorentz

Abstract.

Let $\Lambda_{n}:=\left\{\lambda_{0}<\lambda_{1}<\cdots<\lambda_{n}\right\}$ be a set of real numbers. The collection of all linear combinations of $e^{\lambda_{0} t}, e^{\lambda_{1} t}, \ldots, e^{\lambda_{n} t}$ over $\mathbb{R}$ will be denoted by

$$
E\left(\Lambda_{n}\right):=\operatorname{span}\left\{e^{\lambda_{0} t}, e^{\lambda_{1} t}, \ldots, e^{\lambda_{n} t}\right\} .
$$

Elements of $E\left(\Lambda_{n}\right)$ are called exponential sums of $n+1$ terms. Let $\|f\|_{[a, b]}$ denote the uniform norm of a real valued function $f$ defined on $[a, b]$. We prove the following results.

Theorem 1. Let $n \geq 2$ be an integer. Let $\Lambda_{n}:=\left\{\lambda_{0}<\lambda_{1}<\cdots<\lambda_{n}\right\}$ be a set of nonnegative real numbers, $b \in \mathbb{R}$. Then there is an absolute constant $c_{1}>0$ such that

$$
\frac{c_{1}}{\log n} \sum_{j=0}^{n} \lambda_{j} \leq \sup _{P} \frac{\left\|P^{\prime}\right\|_{(-\infty, b]}}{\|P\|_{(-\infty, b]}} \leq 9 \sum_{j=0}^{n} \lambda_{j},
$$

where the supremum is taken for all $0 \neq P \in E\left(\Lambda_{n}\right)$ increasing on $(-\infty, \infty)$.

Theorem 2. Let $n \geq 2$ be an integer. Let $\Lambda_{n}:=\left\{\lambda_{0}<\lambda_{1}<\cdots<\lambda_{n}\right\}$ be a set of real numbers. Let $[a, b]$ be a finite interval with length $b-a>0$. There are positive constants $c_{2}=c_{2}(a, b)$ and $c_{3}=c_{3}(a, b)$ depending only on $a$ and $b$ such that

$$
c_{2}\left(n^{2}+\frac{1}{\log n} \sum_{j=0}^{n}\left|\lambda_{j}\right|\right) \leq \sup _{P} \frac{\left\|P^{\prime}\right\|_{[a, b]}}{\|P\|_{[a, b]}} \leq c_{3}\left(n^{2}+\sum_{j=0}^{n}\left|\lambda_{j}\right|\right),
$$

where the supremum is taken for all $0 \neq P \in E\left(\Lambda_{n}\right)$ increasing on $(-\infty, \infty)$.

It is expected that the factor $1 / \log n$ in the above theorems can be dropped.

\section{Introduction and Notation}

Throughout the paper $[a, b]$ denotes a finite interval of length $b-a>0$.

The Markov inequality asserts that

$$
\left\|p^{\prime}\right\|_{[-1,1]} \leq n^{2}\|p\|_{[-1,1]}
$$

Key words and phrases. Markov inequality, Newman inequality, increasing exponential sums. 2000 Mathematics Subject Classifications: Primary: 41A17 
for all polynomials of degree at most $n$ (with real coefficients). Here, and in what follows $\|f\|_{[a, b]}$ denotes the uniform norm of a real valued function $f$ defined on $[a, b]$.

It has been observed by Bernstein that Markov's inequality for monotone polynomials is not essential better than that for all polynomials. He proved that

$$
\sup _{p} \frac{\left\|p^{\prime}\right\|_{[-1,1]}}{\|p\|_{[-1,1]}}= \begin{cases}\frac{1}{4}(n+1)^{2}, & \text { if } n \text { is odd } \\ \frac{1}{4} n(n+2), & \text { if } n \text { is even }\end{cases}
$$

where the supremum is taken for all polynomials $0 \neq p$ of degree at most $n$ that are monotone on $[-1,1]$. See [16, p. 607], for instance.

In his book [2] Braess writes "The rational functions and exponential sums belong to those concrete families of functions which are the most frequently used in nonlinear approximation theory. The starting point of consideration of exponential sums is an approximation problem often encountered for the analysis of decay processes in natural sciences. A given empirical function on a real interval is to be approximated by sums of the form

$$
\sum_{j=1}^{n} a_{j} e^{\lambda_{j} t}
$$

where the parameters $a_{j}$ and $\lambda_{j}$ are to be determined, while $n$ is fixed."

Let

$$
E_{n}:=\left\{f: f(t)=a_{0}+\sum_{j=1}^{n} a_{j} e^{\lambda_{j} t}, \quad a_{j}, \lambda_{j} \in \mathbb{R}\right\} .
$$

So $E_{n}$ is the collection of all $n+1$ term exponential sums with constant first term. Schmidt [17] proved that there is a constant $c(n)$ depending only on $n$ so that

$$
\left\|f^{\prime}\right\|_{[a+\delta, b-\delta]} \leq c(n) \delta^{-1}\|f\|_{[a, b]}
$$

for every $f \in E_{n}$ and $\delta \in\left(0, \frac{1}{2}(b-a)\right)$. The main result, Theorem 3.2, of [5] shows that Schmidt's inequality holds with $c(n)=2 n-1$. That is,

$$
\sup _{0 \neq f \in E_{n}} \frac{\left|f^{\prime}(y)\right|}{\|f\|_{[a, b]}} \leq \frac{2 n-1}{\min \{y-a, b-y\}}, \quad y \in(a, b) .
$$

In this Bernstein-type inequality even the point-wise factor is sharp up to a multiplicative absolute constant; the inequality

$$
\frac{1}{e-1} \frac{n-1}{\min \{y-a, b-y\}} \leq \sup _{0 \neq f \in E_{n}} \frac{\left|f^{\prime}(y)\right|}{\|f\|_{[a, b]}}, \quad y \in(a, b),
$$

is established by Theorem 3.3 in [5].

Bernstein-type inequalities play a central role in approximation theory via a machinery developed by Bernstein, which turns Bernstein-type inequalities into inverse theorems of approximation. See, for example, the books by Lorentz [14] and by DeVore and Lorentz 
[9]. From (1.1) one can deduce in a standard fashion that if there is a sequence $\left(f_{n}\right)_{n=1}^{\infty}$ of exponential sums with $f_{n} \in E_{n}$ that approximates $g$ on an interval $[a, b]$ uniformly with errors

$$
\left\|g-f_{n}\right\|_{[a, b]}=O\left(n^{-m}(\log n)^{-2}\right), \quad n=2,3, \ldots,
$$

where $m \in \mathbb{N}$ is a fixed integer, then $g$ is $m$ times continuously differentiable on $(a, b)$. Let $\mathcal{P}_{n}$ be the collection of all polynomials of degree at most $n$ with real coefficients. Inequality (1.1) can be extended to $E_{n}$ replaced by

$$
\widetilde{E}_{n}:=\left\{f: f(t)=a_{0}+\sum_{j=1}^{N} P_{m_{j}}(t) e^{\lambda_{j} t}, \quad a_{0}, \lambda_{j} \in \mathbb{R}, \quad P_{m_{j}} \in \mathcal{P}_{m_{j}}, \quad \sum_{j=1}^{N}\left(m_{j}+1\right) \leq n\right\} .
$$

In fact, it is well-known that $\widetilde{E}_{n}$ is the uniform closure of $E_{n}$ on any finite subinterval of the real number line. For a function $f$ defined on a set $A$ let

$$
\|f\|_{A}:=\|f\|_{L_{\infty} A}:=\|f\|_{L_{\infty}(A)}:=\sup _{x \in A}|f(x)|
$$

and let

$$
\|f\|_{L_{p} A}:=\|f\|_{L_{p}(A)}:=\left(\int_{A}|f(x)|^{p} d x\right)^{1 / p}, \quad p>0,
$$

whenever the Lebesgue integral exists.

In this paper we make an effort to show that Newman's type inequality (Theorem 2.1) for exponential sums on $(-\infty, b]$ and its extension to finite intervals $[a, b]$ (the case $p=\infty$ in Theorem 2.3) remain essentially sharp even if we consider only increasing exponential sums on the real number line.

\section{Some Recent Results}

Let $\Lambda_{n}:=\left\{\lambda_{0}<\lambda_{1}<\cdots<\lambda_{n}\right\}$ be a set of real numbers. The collection of all linear combinations of $e^{\lambda_{0} t}, e^{\lambda_{1} t}, \ldots, e^{\lambda_{n} t}$ over $\mathbb{R}$ will be denoted by

$$
E\left(\Lambda_{n}\right):=\operatorname{span}\left\{e^{\lambda_{0} t}, e^{\lambda_{1} t}, \ldots, e^{\lambda_{n} t}\right\}
$$

Elements of $E\left(\Lambda_{n}\right)$ are called exponential sums of $n+1$ terms. Newman's inequality (see [3] and [15]) is an essentially sharp Markov-type inequality for $E\left(\Lambda_{n}\right)$ on $(-\infty, 0]$ in the case when each $\lambda_{j}$ is nonnegative.

Theorem 2.1 (Newman's Inequality). Let $\Lambda_{n}:=\left\{\lambda_{0}<\lambda_{1}<\cdots<\lambda_{n}\right\}$ be a set of nonnegative real numbers. Let $b \in \mathbb{R}$. Then

$$
\frac{2}{3} \sum_{j=0}^{n} \lambda_{j} \leq \sup _{0 \neq P \in E\left(\Lambda_{n}\right)} \frac{\left\|P^{\prime}\right\|_{(-\infty, b]}}{\|P\|_{(-\infty, b]}} \leq 9 \sum_{j=0}^{n} \lambda_{j}
$$

An $L_{p}$ version of this is established in [3], [6], [8], and [10]. 
Theorem 2.2. Let $\Lambda_{n}:=\left\{\lambda_{0}<\lambda_{1}<\cdots<\lambda_{n}\right\}$ be a set of nonnegative real numbers. Let $1 \leq p \leq \infty$. Let $b \in \mathbb{R}$. Then

$$
\left\|P^{\prime}\right\|_{L_{p}(-\infty, b]} \leq 9\left(\sum_{j=0}^{n} \lambda_{j}\right)\|P\|_{L_{p}(-\infty, b]}
$$

for every $P \in E\left(\Lambda_{n}\right)$.

Note that in the above theorems the case $b=0$ represents the general case. This can be seen by the substitution $u=t-b$.

The following $L_{p}[a, b](1 \leq p \leq \infty)$ analog of Theorem 2.2 has been established in [1].

Theorem 2.3. Let $n \geq 1$ be an integer. Let $\Lambda_{n}:=\left\{\lambda_{0}<\lambda_{1}<\cdots<\lambda_{n}\right\}$ be a set of real numbers. Let $1 \leq p \leq \infty$. There is a positive constant $c_{4}=c_{4}(a, b)$ depending only on a and $b$ such that

$$
\sup _{0 \neq P \in E\left(\Lambda_{n}\right)} \frac{\left\|P^{\prime}\right\|_{L_{p}[a, b]}}{\|P\|_{L_{p}[a, b]}} \leq c_{4}\left(n^{2}+\sum_{j=0}^{n}\left|\lambda_{j}\right|\right) .
$$

Theorem 2.3 was proved earlier in [4] and [10] under the additional assumptions that $\lambda_{j} \geq \delta j$ for each $j$ with a constant $\delta>0$ and with $c_{4}=c_{4}(a, b)$ replaced by $c_{4}=c_{4}(a, b, \delta)$ depending only on $a, b$, and $\delta$. The novelty of Theorem 2.3 was the fact that $\Lambda_{n}:=\left\{\lambda_{0}<\right.$ $\left.\lambda_{1}<\cdots<\lambda_{n}\right\}$ is an arbitrary set of real numbers, not even the nonnegativity of the exponents $\lambda_{j}$ is needed.

In [11] the following Nikolskii-Markov type inequality has been proved for $E\left(\Lambda_{n}\right)$ on $(-\infty, 0]$.

Theorem 2.4. Suppose $0<q \leq p \leq \infty$. Let $\Lambda_{n}:=\left\{\lambda_{0}<\lambda_{1}<\cdots<\lambda_{n}\right\}$ be a set of nonnegative real numbers. Let $\mu$ be a nonnegative integer. Let $b \in \mathbb{R}$. There are constants $c_{5}=c_{5}(p, q, \mu)>0$ and $c_{6}=c_{6}(p, q, \mu)>0$ depending only on $p$, $q$, and $\mu$ such that

$$
c_{5}\left(\sum_{j=1}^{n} \lambda_{j}\right)^{\mu+\frac{1}{q}-\frac{1}{p}} \leq \sup _{0 \neq P \in E\left(\Lambda_{n}\right)} \frac{\left\|P^{(\mu)}\right\|_{L_{p}(-\infty, b]}}{\|P\|_{L_{q}(-\infty, b]}} \leq c_{6}\left(\sum_{j=1}^{n} \lambda_{j}\right)^{\mu+\frac{1}{q}-\frac{1}{p}},
$$

where the lower bound holds for all $0<q \leq p \leq \infty$ and for all $\mu \geq 0$, while the upper bound holds when $\mu=0$ and $0<q \leq p \leq \infty$, and when $\mu \geq 1, p \geq 1$, and $0<q \leq p \leq \infty$. Also, there are constants $c_{5}=c_{5}(q, \mu)>0$ and $c_{6}=c_{6}(q, \mu)>0$ depending only on $q$ and $\mu$ such that

$$
c_{5}\left(\sum_{j=1}^{n} \lambda_{j}\right)^{\mu+\frac{1}{q}} \leq \sup _{0 \neq P \in E\left(\Lambda_{n}\right)} \frac{\left|P^{(\mu)}(y)\right|}{\|P\|_{L_{q}(-\infty, y]}} \leq c_{6}\left(\sum_{j=1}^{n} \lambda_{j}\right)^{\mu+\frac{1}{q}}
$$

for every $y \in \mathbb{R}$.

Motivated by a question of Michel Weber (Strasbourg) in [13] we proved the following couple of theorems. 
Theorem 2.5. Let $\Lambda_{n}:=\left\{\lambda_{0}<\lambda_{1}<\cdots<\lambda_{n}\right\}$ be a set of real numbers. Suppose $0<q \leq p \leq \infty$. There are constants $c_{7}=c_{7}(p, q, a, b)>0$ and $c_{8}=c_{8}(p, q, a, b)>0$ depending only on $p, q$, $a$, and $b$ such that

$$
c_{7}\left(n^{2}+\sum_{j=1}^{n}\left|\lambda_{j}\right|\right)^{\frac{1}{q}-\frac{1}{p}} \leq \sup _{0 \neq P \in E\left(\Lambda_{n}\right)} \frac{\|P\|_{L_{p}[a, b]}}{\|P\|_{L_{q}[a, b]}} \leq c_{8}\left(n^{2}+\sum_{j=1}^{n}\left|\lambda_{j}\right|\right)^{\frac{1}{q}-\frac{1}{p}} .
$$

Theorem 2.6. Let $\Lambda_{n}:=\left\{\lambda_{0}<\lambda_{1}<\cdots<\lambda_{n}\right\}$ be a set of real numbers. Suppose $0<q \leq p \leq \infty$. There are constants $c_{9}=c_{9}(p, q, a, b)>0$ and $c_{10}=c_{10}(p, q, a, b)>0$ depending only on $p, q, a$, and $b$ such that

$$
c_{9}\left(n^{2}+\sum_{j=1}^{n}\left|\lambda_{j}\right|\right)^{1+\frac{1}{q}-\frac{1}{p}} \leq \sup _{0 \neq P \in E\left(\Lambda_{n}\right)} \frac{\left\|P^{\prime}\right\|_{L_{p}[a, b]}}{\|P\|_{L_{q}[a, b]}} \leq c_{10}\left(n^{2}+\sum_{j=1}^{n}\left|\lambda_{j}\right|\right)^{1+\frac{1}{q}-\frac{1}{p}},
$$

where the lower bound holds for all $0<q \leq p \leq \infty$, while the upper bound holds when $p \geq 1$ and $0<q \leq p \leq \infty$.

The lower bounds in these inequalities were shown by a method with the Pinkus-Smith Improvement Theorem in the center. We formulate the useful lemmas applied in the proofs of these lower bounds. To emphasize the power of the technique of interpolation, we present the short proofs of these lemmas, versions of which will be used in the proofs of our new results. We also note that essentially sharp Bernstein-type inequalities for linear combinations of shifted Gaussians are proved in [12].

In fact, a closer look at the proof of Theorem 2.6 presented in [13] gives the following results.

Theorem 2.5*. Suppose $0 \leq \lambda_{0}<\lambda_{1}<\cdots<\lambda_{n}, 0<q \leq \infty$. There are constants $c_{7}=c_{7}(q, a, b)>0$ and $c_{8}=c_{8}(q, a, b)>0$ such that

$$
c_{7}\left(n^{2}+\sum_{j=1}^{n} \lambda_{j}\right)^{\frac{1}{q}} \leq \sup _{0 \neq P \in E\left(\Lambda_{n}\right)} \frac{|P(b)|}{\|P\|_{L_{q}[a, b]}} \leq c_{8}\left(n^{2}+\sum_{j=1}^{n} \lambda_{j}\right)^{\frac{1}{q}} .
$$

Theorem 2.5**. Suppose $\lambda_{0}<\lambda_{1}<\cdots<\lambda_{n} \leq 0,0<q \leq \infty$. There are constants $c_{7}=c_{7}(q, a, b)>0$ and $c_{8}=c_{8}(q, a, b)>0$ such that

$$
c_{7}\left(n^{2}+\sum_{j=1}^{n}\left|\lambda_{j}\right|\right)^{\frac{1}{q}} \leq \sup _{0 \neq P \in E\left(\Lambda_{n}\right)} \frac{|P(a)|}{\|P\|_{L_{q}[a, b]}} \leq c_{8}\left(n^{2}+\sum_{j=1}^{n}\left|\lambda_{j}\right|\right)^{\frac{1}{q}} .
$$


Theorem 2.6*. Suppose $0 \leq \lambda_{0}<\lambda_{1}<\cdots<\lambda_{n}, 0<q \leq \infty$. There are constants $c_{9}=c_{9}(q, a, b)>0$ and $c_{10}=c_{10}(q, a, b)>0$ such that

$$
c_{9}\left(n^{2}+\sum_{j=1}^{n} \lambda_{j}\right)^{1+\frac{1}{q}} \leq \sup _{0 \neq P \in E\left(\Lambda_{n}\right)} \frac{\left|P^{\prime}(b)\right|}{\|P\|_{L_{q}[a, b]}} \leq c_{10}\left(n^{2}+\sum_{j=1}^{n}\left|\lambda_{j}\right|\right)^{1+\frac{1}{q}} .
$$

Theorem 2.6**. Suppose $\lambda_{0}<\lambda_{1}<\cdots<\lambda_{n} \leq 0,0<q \leq \infty$. There are constants $c_{9}=c_{9}(q, a, b)>0$ and $c_{10}=c_{10}(q, a, b)>0$ such that

$$
c_{9}\left(n^{2}+\sum_{j=1}^{n}\left|\lambda_{j}\right|\right)^{1+\frac{1}{q}} \leq \sup _{0 \neq P \in E\left(\Lambda_{n}\right)} \frac{\left|P^{\prime}(a)\right|}{\|P\|_{L_{q}[a, b]}} \leq c_{10}\left(n^{2}+\sum_{j=1}^{n}\left|\lambda_{j}\right|\right)^{1+\frac{1}{q}} .
$$

\section{NeW Results}

We make an effort to show that Newman's inequality (Theorem 2.1) on $(-\infty, b]$ and its extension to finite intervals $[a, b]$ with length $b-a>0$ (the case $p=\infty$ in Theorem 2.3) remain essentially sharp even if we consider only increasing exponential sums on the real number line.

Theorem 3.1. Let $n \geq 2$ be an integer. Let $\Lambda_{n}:=\left\{\lambda_{0}<\lambda_{1}<\cdots<\lambda_{n}\right\}$ be a set of positive real numbers, $b \in \mathbb{R}$. Then there is an absolute constant $c_{1}>0$ such that

$$
\frac{c_{1}}{\log n} \sum_{j=0}^{n} \lambda_{j} \leq \sup _{P} \frac{\left\|P^{\prime}\right\|_{(-\infty, b]}}{\|P\|_{(-\infty, b]}} \leq 9 \sum_{j=0}^{n} \lambda_{j},
$$

where the supremum is taken for all $0 \neq P \in E\left(\Lambda_{n}\right)$ increasing on $(-\infty, \infty)$.

Theorem 3.2. Let $n \geq 2$ be an integer. Let $\Lambda_{n}:=\left\{\lambda_{0}<\lambda_{1}<\cdots<\lambda_{n}\right\}$ be a set of real numbers. There are positive constants $c_{2}=c_{2}(a, b)$ and $c_{3}=c_{3}(a, b)$ depending only on $a$ and $b$ such that

$$
c_{2}\left(n^{2}+\frac{1}{\log n} \sum_{j=0}^{n}\left|\lambda_{j}\right|\right) \leq \sup _{P} \frac{\left\|P^{\prime}\right\|_{[a, b]}}{\|P\|_{[a, b]}} \leq c_{3}\left(n^{2}+\sum_{j=0}^{n}\left|\lambda_{j}\right|\right),
$$

where the supremum is taken for all $0 \neq P \in E\left(\Lambda_{n}\right)$ increasing on $(-\infty, \infty)$.

It is expected that the factor $1 / \log n$ in the above theorems can be dropped.

\section{LEMMAS}

Let $q \in(0, \infty]$ and let $w$ be a not identically zero continuous function defined on $[a, b]$. Our first lemma can be proved by a simple compactness argument and may be viewed as a simple exercise. 
Lemma 4.1. Let $\Delta_{n}:=\left\{\delta_{0}<\delta_{1}<\cdots<\delta_{n}\right\}$ be a set of real numbers. Let $c \in[b, \infty)$. Then there exists a $0 \neq T \in E\left(\Delta_{n}\right)$ such that

$$
\frac{|T(c)|}{\|T w\|_{L_{q}[a, b]}}=\sup _{0 \neq P \in E\left(\Delta_{n}\right)} \frac{|P(c)|}{\|P w\|_{L_{q}[a, b]}}
$$

and there exists a $0 \neq S \in E\left(\Delta_{n}\right)$ such that

$$
\frac{\left|S^{\prime}(c)\right|}{\|S w\|_{L_{q}[a, b]}}=\sup _{0 \neq P \in E\left(\Delta_{n}\right)} \frac{\left|P^{\prime}(c)\right|}{\|P w\|_{L_{q}[a, b]}} .
$$

Our next lemma is an essential tool in proving our key lemmas, Lemmas 4.3 and 4.4.

Lemma 4.2. Let $\Delta_{n}:=\left\{\delta_{0}<\delta_{1}<\cdots<\delta_{n}\right\}$ be a set of real numbers. Let $c \in(b, \infty)$. Let $T$ and $S$ be the same as in Lemma 4.1. Then $T$ has exactly $n$ zeros in $[a, b]$ by counting multiplicities. Under the additional assumption $\delta_{n} \geq 0, S$ also has exactly $n$ zeros in $[a, b]$ by counting multiplicities.

The heart of the proof of our theorems is the following pair of comparison lemmas. The proof of the next couple of lemmas is based on basic properties of Descartes systems, in particular on Descartes' Rule of Sign, and on a technique used earlier by P.W. Smith and Pinkus. Lorentz ascribes this result to Pinkus, although it was P.W. Smith [18] who published it. I have learned about the the method of proofs of these lemmas from Peter Borwein, who also ascribes it to Pinkus. This is the proof we present here. Section 3.2 of [3], for instance, gives an introduction to Descartes systems. Descartes' Rule of Signs is stated and proved on page 102 of [3].

Lemma 4.3. Let $\Delta_{n}:=\left\{\delta_{0}<\delta_{1}<\cdots<\delta_{n}\right\}$ and $\Gamma_{n}:=\left\{\gamma_{0}<\gamma_{1}<\cdots<\gamma_{n}\right\}$ be sets of real numbers satisfying $\delta_{j} \leq \gamma_{j}$ for each $j=0,1, \ldots, n$. Let $c \in[b, \infty)$. Then

$$
\sup _{0 \neq P \in E\left(\Delta_{n}\right)} \frac{|P(c)|}{\|P w\|_{L_{q}[a, b]}} \leq \sup _{0 \neq P \in E\left(\Gamma_{n}\right)}\left\{\frac{|P(c)|}{\|P w\|_{L_{q}[a, b]}}\right\} .
$$

Under the additional assumption $\delta_{n} \geq 0$ we also have

$$
\sup _{0 \neq P \in E\left(\Delta_{n}\right)} \frac{\left|P^{\prime}(c)\right|}{\|P w\|_{L_{q}[a, b]}} \leq \sup _{0 \neq P \in E\left(\Gamma_{n}\right)} \frac{\left|P^{\prime}(c)\right|}{\|P w\|_{L_{q}[a, b]}} .
$$

In addition, the above inequalities hold if the supremums are taken over all nonnegative not identically zero $P \in E\left(\Delta_{n}\right)$ and $P \in E\left(\Gamma_{n}\right)$, respectively.

The result below follows from Lemma 4.3 by a standard compactness argument.

Lemma 4.3*. The statements of Lemma 4.3 remain valid if $\delta_{0}>0$, the interval $[a, b]$ is replaced by $(-\infty, b]$, and $w$ is a not identically zero, continuous, and bounded function on on $(-\infty, b]$. 
Lemma 4.4. Let $\Delta_{n}:=\left\{\delta_{0}<\delta_{1}<\cdots<\delta_{n}\right\}$ and $\Gamma_{n}:=\left\{\gamma_{0}<\gamma_{1}<\cdots<\gamma_{n}\right\}$ be sets of real numbers satisfying $\delta_{j} \leq \gamma_{j}$ for each $j=0,1, \ldots, n$. Let $c \in(-\infty, a]$. Then

$$
\sup _{0 \neq P \in E\left(\Delta_{n}\right)} \frac{|P(c)|}{\|P w\|_{L_{q}[a, b]}} \geq \sup _{0 \neq P \in E\left(\Gamma_{n}\right)} \frac{|P(c)|}{\|P w\|_{L_{q}[a, b]}} .
$$

Under the additional assumption $\gamma_{0} \leq 0$ we also have

$$
\sup _{0 \neq P \in E\left(\Delta_{n}\right)} \frac{\left|Q^{\prime}(c)\right|}{\|Q w\|_{L_{q}[a, b]}} \geq \sup _{0 \neq P \in E\left(\Gamma_{n}\right)} \frac{\left|Q^{\prime}(c)\right|}{\|Q w\|_{L_{q}[a, b]}} .
$$

In addition, the above inequalities hold if the supremums are taken over all nonnegative not identically zero $P \in E\left(\Delta_{n}\right)$ and $P \in E\left(\Gamma_{n}\right)$, respectively.

The result below follows from Lemma 4.4 by a standard compactness argument.

Lemma 4.4*. The statements of Lemma 4.4 remain valid if $\gamma_{n}<0$, the interval $[a, b]$ is replaced by $[a, \infty)$, and $w$ is a not identically zero, continuous, and bounded function on $[a, \infty)$.

\section{Proofs of the Lemmas}

Proof of Lemma 4.1. Since $\Delta_{n}$ is fixed, the proof is a standard compactness argument. We omit the details.

To prove Lemma 4.2 we need the following two facts. (a) Every $0 \neq f \in E\left(\Delta_{n}\right)$ has at most $n$ real zeros by counting multiplicities. (b) If $t_{1}<t_{2}<\cdots<t_{m}$ are real numbers and $k_{1}, k_{2}, \ldots, k_{m}$ are positive integers such that $\sum_{j=1}^{m} k_{j}=n$, then there is a $0 \neq f \in E\left(\Delta_{n}\right)$ having a zero at $t_{j}$ with multiplicity $k_{j}$ for each $j=1,2, \ldots, m$.

Proof of Lemma 4.2. We prove the statement for $T$ first. Suppose to the contrary that $t_{1}<t_{2}<\cdots<t_{m}$ are real numbers in $[a, b]$ such that $t_{j}$ is a zero of $T$ with multiplicity $k_{j}$ for each $j=1,2, \ldots, m, k:=\sum_{j=1}^{m} k_{j}<n$, and $T$ has no other zeros in $[a, b]$ different from $t_{1}, t_{2}, \ldots, t_{m}$. Let $t_{m+1}:=c$ and $k_{m+1}:=n-k \geq 1$. Choose an $0 \neq R \in E\left(\Delta_{n}\right)$ such that $R$ has a zero at $t_{j}$ with multiplicity $k_{j}$ for each $j=1,2, \ldots, m+1$, and normalize so that $T(t)$ and $R(t)$ have the same sign at every $t \in[a, b]$. Let $T_{\varepsilon}:=T-\varepsilon R$. Note that $T$ and $R$ are of the form

$$
T(t)=\widetilde{T}(t) \prod_{j=1}^{m}\left(t-t_{j}\right)^{k_{j}} \quad \text { and } \quad R(t)=\widetilde{R}(t) \prod_{j=1}^{m}\left(t-t_{j}\right)^{k_{j}}
$$

where both $\widetilde{T}$ and $\widetilde{R}$ are continuous functions on $[a, b]$ having no zeros on $[a, b]$. Hence, if $\varepsilon>0$ is sufficiently small, then $\left|T_{\varepsilon}(t)\right|<|T(t)|$ at every $t \in[a, b] \backslash\left\{t_{1}, t_{2}, \ldots, t_{m}\right\}$, so

$$
\left\|T_{\varepsilon} w\right\|_{L_{q}[a, b]}<\|T w\|_{L_{q}[a, b]} .
$$

This, together with $T_{\varepsilon}(c)=T(c)$, contradicts the maximality of $T$. 
Now we prove the statement for $S$. Without loss of generality we may assume that $S^{\prime}(c)>0$. Suppose to the contrary that $t_{1}<t_{2}<\cdots<t_{m}$ are real numbers in $[a, b]$ such that $t_{j}$ is a zero of $S$ with multiplicity $k_{j}$ for each $j=1,2, \ldots, m, k:=\sum_{j=1}^{m} k_{j}<n$, and $S$ has no other zeros in $[a, b]$ different from $t_{1}, t_{2}, \ldots, t_{m}$. Choose a

$$
0 \neq Q \in \operatorname{span}\left\{e^{\delta_{n-k} t}, e^{\delta_{n-k+1} t}, \ldots, e^{\delta_{n} t}\right\} \subset E\left(\Delta_{n}\right)
$$

such that $Q$ has a zero at $t_{j}$ with multiplicity $k_{j}$ for each $j=1,2, \ldots, m$, and normalize so that $S(t)$ and $Q(t)$ have the same sign at every $t \in[a, b]$. Note that $S$ and $Q$ are of the form

$$
S(t)=\widetilde{S}(t) \prod_{j=1}^{m}\left(t-t_{j}\right)^{k_{j}} \quad \text { and } \quad Q(t)=\widetilde{Q}(t) \prod_{j=1}^{m}\left(t-t_{j}\right)^{k_{j}},
$$

where both $\widetilde{S}$ and $\widetilde{Q}$ are continuous functions on $[a, b]$ having no zeros on $[a, b]$. Let $t_{m+1}:=c$ and $k_{m+1}:=1$. Choose an

$$
0 \neq R \in \operatorname{span}\left\{e^{\delta_{n-k-1} t}, e^{\delta_{n-k} t}, \ldots, e^{\delta_{n} t}\right\} \subset E\left(\Delta_{n}\right)
$$

such that $R$ has a zero at $t_{j}$ with multiplicity $k_{j}$ for each $j=1,2, \ldots, m+1$, and normalize so that $S(t)$ and $R(t)$ have the same sign at every $t \in[a, b]$. Note that $S$ and $R$ are of the form

$$
S(t)=\widetilde{S}(t) \prod_{j=1}^{m}\left(t-t_{j}\right)^{k_{j}} \quad \text { and } \quad R(t)=\widetilde{R}(t) \prod_{j=1}^{m}\left(t-t_{j}\right)^{k_{j}}
$$

where both $\widetilde{S}$ and $\widetilde{R}$ are continuous functions on $[a, b]$ having no zeros on $[a, b]$. It can be easily seen that $\delta_{n} \geq 0$ implies that $Q^{\prime}(t)$ does not vanish on $\left(t_{m}, \infty\right)$ (divide by $e^{\delta_{n} t}$ and then use Rolle's Theorem). Similarly, since $\delta_{n} \geq 0$, it is easy to see that if $Q^{\prime}$ is positive on $\left(t_{m}, \infty\right)$, then $R^{\prime}$ is negative on $(c, \infty)$. Hence $Q^{\prime}(c) R^{\prime}(c)<0$, so the sign of $Q^{\prime}(c)$ is different from the sign of $R^{\prime}(c)$. Let $U:=Q$ if $Q^{\prime}(c)<0$ and let $U:=R$ if $R^{\prime}(c)<0$. Let $S_{\varepsilon}:=S-\varepsilon U$. Hence, if $\varepsilon>0$ is sufficiently small, then $\left|S_{\varepsilon}(t)\right|<|T(t)|$ at every $t \in[a, b] \backslash\left\{t_{1}, t_{2}, \ldots, t_{m}\right\}$, so

$$
\left\|S_{\varepsilon} w\right\|_{L_{q}[a, b]}<\|S w\|_{L_{q}[a, b]} .
$$

This, together with $S_{\varepsilon}^{\prime}(c)>S^{\prime}(c)>0$, contradicts the maximality of $S$.

Proof of Lemma 4.3. We begin with the first inequality. We may assume that $a<b<c$. The general case when $a<b \leq c$ follows by a standard continuity argument. Let $k \in$ $\{0,1, \ldots, n\}$ be fixed and let

$$
\gamma_{0}<\gamma_{1}<\cdots<\gamma_{n}, \quad \gamma_{j}=\delta_{j}, \quad j \neq k, \quad \text { and } \quad \delta_{k}<\gamma_{k}<\delta_{k+1}
$$

(let $\delta_{n+1}:=\infty$ ). To prove the lemma it is sufficient to study the above cases since the general case follows from this by a finite number of pairwise comparisons. By Lemmas 4.1 and 4.2 , there is a $0 \neq T \in E\left(\Delta_{n}\right)$ such that

$$
\frac{|T(c)|}{\|T w\|_{L_{q}[a, b]}}=\sup _{0 \neq P \in E\left(\Delta_{n}\right)} \frac{|P(c)|}{\|P w\|_{L_{q}[a, b]}},
$$


where $T$ has exactly $n$ zeros in $[a, b]$ by counting multiplicities. Denote the distinct zeros of $T$ in $[a, b]$ by $t_{1}<t_{2}<\cdots<t_{m}$, where $t_{j}$ is a zero of $T$ with multiplicity $k_{j}$ for each $j=1,2, \ldots, m$, and $\sum_{j=1}^{m} k_{j}=n$. Then $T$ has no other zeros in $\mathbb{R}$ different from $t_{1}, t_{2}, \ldots, t_{m}$. Let

$$
T(t)=: \sum_{j=0}^{n} a_{j} e^{\delta_{j} t}, \quad a_{j} \in \mathbb{R} .
$$

Without loss of generality we may assume that $T(c)>0$. We have $T(t)>0$ for every $t>c$, otherwise, in addition to its $n$ zeros in $[a, b]$ (by counting multiplicities), $T$ would have at least one more zero in $(c, \infty)$, which is impossible. Hence

$$
a_{n}:=\lim _{t \rightarrow \infty} T(t) e^{-\delta_{n} t} \geq 0
$$

Since $E\left(\Delta_{n}\right)$ is the span of a Descartes system on $(-\infty, \infty)$, it follows from Descartes' Rule of Signs that

$$
(-1)^{n-j} a_{j}>0, \quad j=0,1, \ldots, n .
$$

So, in particular, $a_{n}>0$. Choose $R \in E\left(\Gamma_{n}\right)$ of the form

$$
R(t)=\sum_{j=0}^{n} b_{j} e^{\gamma_{j} t}, \quad b_{j} \in \mathbb{R}
$$

so that $R$ has a zero at each $t_{j}$ with multiplicity $k_{j}$ for each $j=1,2, \ldots, m$, and normalize so that $R(c)=T(c)(>0)$ (this $R \in E\left(\Gamma_{n}\right)$ is uniquely determined). Similarly to $a_{n} \geq 0$ we have $b_{n} \geq 0$. Since $E\left(\Gamma_{n}\right)$ is the span of a Descartes system on $(-\infty, \infty)$, Descartes' Rule of Signs yields,

$$
(-1)^{n-j} b_{j}>0, \quad j=0,1, \ldots, n .
$$

So, in particular, $b_{n}>0$. We have

$$
(T-R)(t)=a_{k} e^{\delta_{k} t}-b_{k} e^{\gamma_{k} t}+\sum_{\substack{j=0 \\ j \neq k}}^{n}\left(a_{j}-b_{j}\right) e^{\delta_{j} t}
$$

Since $T-R$ has altogether at least $n+1$ zeros at $t_{1}, t_{2}, \ldots, t_{m}$, and $c$ (by counting multiplicities), it does not have any zero on $\mathbb{R}$ different from $t_{1}, t_{2}, \ldots, t_{m}$, and $c$. Since

$$
\left(e^{\delta_{0} t}, e^{\delta_{1} t}, \ldots, e^{\delta_{k} t}, e^{\gamma_{k} t}, e^{\delta_{k+1} t}, \ldots, e^{\delta_{n} t}\right)
$$

is a Descartes system on $(-\infty, \infty)$, Descartes' Rule of Signs implies that the sequence

$$
\left(a_{0}-b_{0}, a_{1}-b_{1}, \ldots, a_{k-1}-b_{k-1}, a_{k},-b_{k}, a_{k+1}-b_{k+1}, \ldots, a_{n}-b_{n}\right)
$$

strictly alternates in sign. Since $(-1)^{n-k} a_{k}>0$, this implies that $a_{n}-b_{n}<0$ if $k<n$, and $-b_{n}<0$ if $k=n$, so

$$
(T-R)(t)<0, \quad t>c .
$$


This can be seen by dividing the left hand side by $e^{\gamma_{n} t}$ and taking the limit as $t \rightarrow \infty$. Since each of $T, R$, and $T-R$ has a zero at $t_{j}$ with multiplicity $k_{j}$ for each $j=1,2, \ldots, m$; $\sum_{j=1}^{m} k_{j}=n$, and $T-R$ has a sign change (a zero with multiplicity 1 ) at $c$, we can deduce that each of $T, R$, and $T-R$ has the same sign on each of the intervals $\left(t_{j}, t_{j+1}\right)$ for every $j=0,1, \ldots, m$ with $t_{0}:=-\infty$ and $t_{m+1}:=c$. Hence $|R(t)| \leq|T(t)|$ holds for all $t \in[a, b] \subset[a, c]$ with strict inequality at every $t$ different from $t_{1}, t_{2}, \ldots, t_{m}$. Combining this with $R(c)=T(c)$, we obtain

$$
\frac{|R(c)|}{\|R w\|_{L_{q}[a, b]}} \geq \frac{|T(c)|}{\|T w\|_{L_{q}[a, b]}}=\sup _{0 \neq P \in E\left(\Delta_{n}\right)} \frac{|P(c)|}{\|P w\|_{L_{q}[a, b]}} .
$$

Since $R \in E\left(\Gamma_{n}\right)$, the first conclusion of the lemma follows from this.

Now we start the proof of the second inequality of the lemma. Although it is quite similar to that of the first inequality, we present the details. We may assume that $a<b<c$ and $\delta_{n}>0$. The general case when $a<b \leq c$ and $\delta_{n} \geq 0$ follows by a standard continuity argument. Let $k \in\{0,1, \ldots, n\}$ be fixed and let

$$
\gamma_{0}<\gamma_{1}<\cdots<\gamma_{n}, \quad \gamma_{j}=\delta_{j}, \quad j \neq k, \quad \text { and } \quad \delta_{k}<\gamma_{k}<\delta_{k+1}
$$

(let $\delta_{n+1}:=\infty$ ). To prove the lemma it is sufficient to study the above cases since the general case follows from this by a finite number of pairwise comparisons. By Lemmas 4.1 and 4.2 , there is an $0 \neq S \in E\left(\Delta_{n}\right)$ such that

$$
\frac{\left|S^{\prime}(c)\right|}{\|S w\|_{L_{q}[a, b]}}=\sup _{0 \neq P \in E\left(\Delta_{n}\right)} \frac{\left|P^{\prime}(c)\right|}{\|P w\|_{L_{q}[a, b]}},
$$

where $S$ has exactly $n$ zeros in $[a, b]$ by counting multiplicities. Denote the distinct zeros of $S$ in $[a, b]$ by $t_{1}<t_{2}<\cdots<t_{m}$, where $t_{j}$ is a zero of $S$ with multiplicity $k_{j}$ for each $j=1,2, \ldots, m$, and $\sum_{j=1}^{m} k_{j}=n$. Then $S$ has no other zeros in $\mathbb{R}$ different from $t_{1}, t_{2}, \ldots, t_{m}$. Let

$$
S(t)=: \sum_{j=0}^{n} a_{j} e^{\delta_{j} t}, \quad a_{j} \in \mathbb{R} .
$$

Without loss of generality we may assume that $S(c)>0$. Since $\delta_{n}>0$, we have $\lim _{t \rightarrow \infty} S(t)=\infty$, otherwise, in addition to its $n$ zeros in $(a, b), S$ would have at least one more zero in $(c, \infty)$, which is impossible.

Because of the extremal property of $S, S^{\prime}(c) \neq 0$. We show that $S^{\prime}(c)>0$. To see this observe that Rolle's Theorem implies that $S^{\prime} \in E\left(\Delta_{n}\right)$ has at least $n-1$ zeros in $\left[t_{1}, t_{m}\right]$ (by counting multiplicities). If $S^{\prime}(c)<0$, then $S\left(t_{m}\right)=0$ and $\lim _{t \rightarrow \infty} S(t)=\infty$ imply that $S^{\prime}$ has at least 2 more zeros in $\left(t_{m}, \infty\right)$. Thus $S^{\prime}(c)<0$ would imply that $S^{\prime}$ has at least $n+1$ zeros in $[a, \infty)$, which is impossible. Hence $S^{\prime}(c)>0$, indeed. Also $a_{n}:=\lim _{t \rightarrow \infty} S(t) e^{-\delta_{n} t} \geq 0$. Since $E\left(\Delta_{n}\right)$ is the span of a Descartes system on $(-\infty . \infty)$, it follows from Descartes' Rule of Signs that

$$
(-1)^{n-j} a_{j}>0,{ }_{11} j=0,1, \ldots, n .
$$


So, in particular, $a_{n}>0$. Choose $R \in E\left(\Gamma_{n}\right)$ of the form

$$
R(t)=\sum_{j=0}^{n} b_{j} e^{\gamma_{j} t}, \quad b_{j} \in \mathbb{R}
$$

so that $R$ has a zero at each $t_{j}$ with multiplicity $k_{j}$ for each $j=1,2, \ldots, m$, and normalize so that $R(c)=S(c)(>0)$ (this $R \in E\left(\Gamma_{n}\right)$ is uniquely determined). Similarly to $a_{n} \geq 0$ we have $b_{n} \geq 0$. Since $E\left(\Gamma_{n}\right)$ is the span of a Descartes system on $[a, b]$, Descartes' Rule of Signs implies that

$$
(-1)^{n-j} b_{j}>0, \quad j=0,1, \ldots, n .
$$

So, in particular, $b_{n}>0$. We have

$$
(S-R)(t)=a_{k} e^{\delta_{k} t}-b_{k} e^{\gamma_{k} t}+\sum_{\substack{j=0 \\ j \neq k}}^{n}\left(a_{j}-b_{j}\right) e^{\delta_{j} t} .
$$

Since $S-R$ has altogether at least $n+1$ zeros at $t_{1}, t_{2}, \ldots, t_{m}$, and $c$ (by counting multiplicities), it does not have any zero on $\mathbb{R}$ different from $t_{1}, t_{2}, \ldots, t_{m}$, and $c$. Since

$$
\left(e^{\delta_{0} t}, e^{\delta_{1} t}, \ldots, e^{\delta_{k} t}, e^{\gamma_{k} t}, e^{\delta_{k+1} t}, \ldots, e^{\delta_{n} t}\right)
$$

is a Descartes system on $(-\infty, \infty)$, Descartes' Rule of Signs implies that the sequence

$$
\left(a_{0}-b_{0}, a_{1}-b_{1}, \ldots, a_{k-1}-b_{k-1}, a_{k},-b_{k}, a_{k+1}-b_{k+1}, \ldots, a_{n}-b_{n}\right)
$$

strictly alternates in sign. Since $(-1)^{n-k} a_{k}>0$, this implies that $a_{n}-b_{n}<0$ if $k<n$ and $-b_{n}<0$ if $k=n$, so

$$
(S-R)(t)<0, \quad t>c .
$$

Since each of $S, R$, and $S-R$ has a zero at $t_{j}$ with multiplicity $k_{j}$ for each $j=1,2, \ldots, m$; $\sum_{j=1}^{m} k_{j}=n$, and $S-R$ has a sign change (a zero with multiplicity 1 ) at $c$, we can deduce that each of $S, R$, and $S-R$ has the same sign on each of the intervals $\left(t_{j}, t_{j+1}\right)$ for every $j=0,1, \ldots, m$ with $t_{0}:=-\infty$ and $t_{m+1}:=c$. Hence $|R(t)| \leq|S(t)|$ holds for all $t \in[a, b] \subset[a, c]$ with strict inequality at every $t$ different from $t_{1}, t_{2}, \ldots, t_{m}$. Combining this with $0<S^{\prime}(c) \leq R^{\prime}(c)$ (recall that $R(c)=S(c)>0$ ), we obtain

$$
\frac{\left|R^{\prime}(c)\right|}{\|R w\|_{L_{q}[a, b]}} \geq \frac{\left|S^{\prime}(c)\right|}{\|S w\|_{L_{q}[a, b]}}=\sup _{0 \neq P \in E\left(\Delta_{n}\right)} \frac{\left|P^{\prime}(c)\right|}{\|P w\|_{L_{q}[a, b]}} .
$$

Since $R \in E\left(\Gamma_{n}\right)$, the second conclusion of the lemma follows from this. The proof of the last statement of the lemma is very similar. We omit the details.

Proof of Lemma 4.4. The lemma follows from Lemma 4.3 by the substitution $u=-t$. 


\section{Proofs of the Theorems}

Proof of Theorem 3.1. In the light of Theorem 2.1 we need to prove only the lower bound. Moreover, it is sufficient to prove only that for every $a<b$ there is a $0 \neq Q \in E\left(\Lambda_{n}\right)$ increasing on $(-\infty, \infty)$ such that

$$
\frac{\left|Q^{\prime}(b)\right|}{\|Q\|_{[a, b]}} \geq \frac{c_{1}}{\log n} \sum_{j=0}^{n} \lambda_{j}
$$

with an absolute constant $c_{1}>0$, and the lower bound of the theorem follows by a standard compactness argument. Let

$$
\lambda:=\frac{1}{3 \log n} \sum_{j=0}^{n} \lambda_{j}
$$

Then there is a $k \in\{0,1, \ldots, n\}$ such that

$$
\lambda_{k} \geq \frac{\lambda}{n-k+1}
$$

Let $\varepsilon>0, m:=\left\lfloor\frac{n-k}{2}\right\rfloor$,

$$
\widetilde{\delta}_{j}:=\frac{\lambda}{2(n-k+1)}+j \varepsilon, \quad j=0,1, \ldots, m
$$

Let $\widetilde{\Delta}_{m}:=\left\{\widetilde{\delta}_{0}<\widetilde{\delta}_{1}<\cdots<\widetilde{\delta}_{m}\right\}$. By Theorem 2.4 there is a $0 \neq R_{m} \in E\left(\widetilde{\Delta}_{m}\right)$ such that

$$
\frac{\left|R_{m}(b)\right|}{\left\|R_{m}\right\|_{L_{2}[a, b]}} \geq \frac{\left|R_{m}(b)\right|}{\left\|R_{m}\right\|_{L_{2}(-\infty, b]}} \geq c_{5}\left(\sum_{j=0}^{m} \widetilde{\delta}_{j}\right)^{1 / 2} \geq c_{5}\left(\frac{(m+1) \lambda}{2(n-k+1)}\right)^{1 / 2} \geq \frac{c_{5}}{2} \lambda^{1 / 2}
$$

Moreover, by Lemma 4.2 we may assume that $R_{m}$ has $m$ zeros in $[a, b]$. Now let

$$
\begin{gathered}
\gamma_{j}:=\lambda_{j+k} \quad \text { and } \quad \delta_{j}:=\frac{\lambda}{n-k+1}+j \varepsilon, \quad j=0,1, \ldots, 2 m, \\
\Delta_{2 m}:=\left\{\delta_{0}<\delta_{1}<\cdots<\delta_{2 m}\right\} \quad \text { and } \quad \Gamma_{2 m}:=\left\{\gamma_{0}<\gamma_{1}<\cdots<\gamma_{2 m}\right\} .
\end{gathered}
$$

Then

$$
P_{2 m}=R_{m}^{2} \in E\left(\Delta_{2 m}\right)
$$

is nonnegative on $(-\infty, \infty)$ having $2 m$ zeros in $(-\infty, b]$ by counting multiplicities. Now, by Lemma $4.3^{*}$ (if $\varepsilon>0$ is sufficiently small, then the assumptions are satisfied) there is a $0 \neq Q_{2 m} \in E\left(\Gamma_{2 m}\right) \subset E\left(\Lambda_{n}\right)$ such that

$$
\frac{\left|Q_{2 m}(b)\right|}{\left\|Q_{2 m}\right\|_{L_{1}(-\infty, b]}} \geq \frac{\left|P_{2 m}(b)\right|}{\left\|P_{2 m}\right\|_{L_{1}(-\infty, b]}}=\frac{\left|R_{m}(b)\right|^{2}}{\left\|R_{m}\right\|_{L_{2}(-\infty, b]}^{2}} \geq \frac{c_{5}^{2}}{4} \lambda .
$$


Now let $S_{2 m} \in E\left(\Gamma_{2 m}\right) \subset E\left(\Lambda_{n}\right)$ be defined by

$$
S_{2 m}(x)=\int_{-\infty}^{x} Q_{2 m}(t) d t .
$$

Then $S_{2 m}$ is increasing on $(-\infty, \infty)$ and

$$
\frac{\left|S_{2 m}^{\prime}(b)\right|}{\left\|S_{2 m}\right\|_{[a, b]}} \geq \frac{\left|S_{2 m}^{\prime}(b)\right|}{\left\|S_{2 m}\right\|_{(-\infty, b]}} \geq \frac{\left|Q_{2 m}(b)\right|}{\left\|Q_{2 m}\right\|_{L_{1}(-\infty, b]}} \geq \frac{c_{5}^{2}}{4} \lambda .
$$

Note that the constant $c_{5}^{2} / 4$ above is absolute and as such it is independent of $a$ as well. Hence the standard compactness argument in the beginning of the proof can be implemented.

Proof of Theorem 3.2. The upper bound of the theorem follows from Theorem 2.5. Now we turn to the proof of the lower bound. Assume that

$$
\lambda_{0}<\lambda_{1}<\cdots<\lambda_{m}<0 \leq \lambda_{m+1}<\lambda_{m+2}<\cdots<\lambda_{n} .
$$

We distinguish four cases.

Case 1: $\sum_{j=m+1}^{n}\left|\lambda_{j}\right| \geq \frac{1}{2} \sum_{j=0}^{n}\left|\lambda_{j}\right| \geq n^{2} \log n$. In this case the lower bound of Theorem 3.1 gives the lower bound of the theorem.

Case 2: $\sum_{j=0}^{m}\left|\lambda_{j}\right| \geq \frac{1}{2} \sum_{j=0}^{n}\left|\lambda_{j}\right| \geq n^{2} \log n$. In this case the lower bound of Theorem 3.1 gives the lower bound of the theorem after the substitution $u=-t$.

Case 3: $\frac{1}{2} \sum_{j=0}^{n}\left|\lambda_{j}\right| \leq n^{2} \log n$ and $m<n / 2$. Let $k=\lfloor n / 4\rfloor-1$. Without loss of generality we may assume that $n \geq 8$, hence $k \geq 1$. Let

$$
\Delta_{k}:=\left\{\delta_{0}<\delta_{1}<\cdots<\delta_{k}\right\}, \quad \delta_{j}:=j \varepsilon, \quad j=0,1, \ldots, k .
$$

By Theorem $2.5^{*}$ there is an $0 \neq R_{k} \in E\left(\Delta_{k}\right)$ such that

$$
\frac{\left|R_{k}(b)\right|}{\left\|R_{k}\right\|_{L_{2}[a, b]}} \geq c_{7} n .
$$

Moreover, by Lemma 4.2 we may assume that $R_{k}$ has $k$ zeros in $[a, b]$. Now let

$$
\Delta_{2 k}:=\left\{\delta_{0}<\delta_{1}<\cdots<\delta_{2 k}\right\}, \quad \delta_{j}:=j \varepsilon, \quad j=0,1, \ldots, 2 k,
$$

and

$$
\Gamma_{2 k}:=\left\{\gamma_{0}<\gamma_{1}<\cdots<\gamma_{2 k}\right\}:=\left\{\lambda_{n-2 k}<\lambda_{n-2 k+1}<\cdots<\lambda_{n}\right\} .
$$

Then

$$
P_{2 k}=R_{k}^{2} \in E\left(\Delta_{2 k}\right)
$$


is nonnegative on $(-\infty, \infty)$ and has $2 k$ zeros in $[a, b]$ by counting multiplicities. Now, by Lemma $4.3^{*}$ (if $\varepsilon>0$ is sufficiently small, then the assumptions are satisfied) there is a $0 \neq Q_{2 k} \in E\left(\Gamma_{2 k}\right) \subset E\left(\Lambda_{n}\right)$ such that

$$
\frac{\left|Q_{2 k}(b)\right|}{\left\|Q_{2 k}\right\|_{L_{1}[a, b]}} \geq \frac{\left|P_{2 k}(b)\right|}{\left\|P_{2 k}\right\|_{L_{1}[a, b]}}=\frac{\left|R_{k}(b)\right|^{2}}{\left\|R_{k}\right\|_{L_{2}[a, b]}^{2}} \geq c_{7}^{2} n^{2} .
$$

Now let $S_{2 k} \in E\left(\Gamma_{2 k}\right) \subset E\left(\Lambda_{n}\right)$ be defined by

$$
S_{2 k}(x)=\int_{-\infty}^{x} Q_{2 k}(t) d t
$$

Then $S_{2 k}$ is increasing on $(-\infty, \infty)$ and

$$
\frac{\left|S_{2 k}^{\prime}(b)\right|}{\left\|S_{2 k}\right\|_{[a, b]}} \geq \frac{\left|Q_{2 k}(b)\right|}{\left\|Q_{2 k}\right\|_{L_{1}([a, b]}} \geq c_{7}^{2} n^{2} .
$$

Case 4: $\frac{1}{2} \sum_{j=0}^{n}\left|\lambda_{j}\right| \leq n^{2} \log n$ and $m \geq n / 2$. The proof follows from that in Case 3 by the substitution $u=-t$.

\section{REFERENCES}

1. D. Benko, T. Erdélyi, and J. Szabados, The full Markov-Newman inequality for Müntz polynomials on positive intervals, Proc. Amer. Math. Soc. 131 (2003), 2385-2391.

2. D. Braess, Nonlinear Approximation Theory, Springer-Verlag, Berlin, 1986.

3. P. Borwein and T. Erdélyi, Polynomials and Polynomial Inequalities, Springer-Verlag, Graduate Texts in Mathematics, Volume 161, 486 p., New York, NY, 1995.

4. P. Borwein and T. Erdélyi, Newman's inequality for Müntz polynomials on positive intervals, J. Approx. Theory 85 (1996), 132-139.

5. P. Borwein and T. Erdélyi, A sharp Bernstein-type inequality for exponential sums, J. Reine Angew. Math. 476 (1996), 127-141.

6. P. Borwein and T. Erdélyi, The $L_{p}$ version of Newman's inequality for lacunary polynomials, Proc. Amer. Math. Soc. 124 (1996), 101-109.

7. P. Borwein and T. Erdélyi, Pointwise Remez- and Nikolskii-type inequalities for exponential sums, Math. Ann. 316 (2000), 39-60.

8. P. Borwein, T. Erdélyi, and J. Zhang, Müntz systems and orthogonal Müntz-Legendre polynomials, Trans. Amer. Math. Soc. 342 (1994), 523-542.

9. R. A. DeVore and G. G. Lorentz, Constructive Approximation, Springer-Verlag, Berlin, 1993.

10. T. Erdélyi, Markov- and Bernstein-type inequalities for Müntz polynomials and exponential sums in $L_{p}$, J. Approx. Theory 104 (2000), 142-152.

11. T. Erdélyi, Extremal properties of the derivatives of the Newman polynomials, Proc. Amer. Math. Soc. 131 (2003), 3129-3134.

12. T. Erdélyi, Bernstein-type inequalities for linear combinations of shifted Gaussians, Bull. London Math. Soc. 38 (2006), 124-138. 
13. T. Erdélyi, Markov-Nikolskii type inequalities for exponential sums on finite intervals, Adv. Math. 208 (2007), 135-146.

14. G.G. Lorentz, Approximation of Functions, 2nd ed., Chelsea, New York, N.Y., 1986.

15. D. J. Newman, Derivative bounds for Müntz polynomials, J. Approx. Theory 18 (1976), 360-362.

16. Q. I. Rahman and G. Schmeisser, Analytic Theory of Polynomials, Clarendon Press, Oxford, 2002 .

17. E. Schmidt, Zur Kompaktheit der Exponentialsummen, J. Approx. Theory 3 (1970), $445-459$.

18. P. W. Smith, An improvement theorem for Descartes systems, Proc. Amer. Math. Soc. 70 (1978), 26-30.

Department of Mathematics, Texas A\&M University, College Station, Texas 77843, USA

E-mail address: terdelyi@math.tamu.edu 